further evidence against an ischaemic aetiology. Treatment options for this syndrome include negatively chronotropic medications and patient education to avoid the heart rate exceeding the threshold at which LBBB develops. However, this was not feasible in our case due to a resting bradycardia and the condition occurring at rest. Theoretically, using HBP to correct LBBB appears counterintuitive, as it is pacing proximal to the point of abnormal conduction. Despite this, it has a proven success rate in the treatment of LBBB [8] and does appear to be a management option that can offer a striking resolution of symptoms in select patients with this unusual and still poorly understood condition.

Conflict of Interest None

\section{REWORKING THE POST-COVID WAITING LIST - THE PATIENT EXPERIENCE OF IMPLANTABLE LOOP RECORDER EXPLANTATION}

Rahul Chattopadhyay, Mina Fares, Mrinal Thakur, Priyadarshini Bhattacharjee, Julie Hayes, Panagiota Anna Chousou, Peter J Pugh. Cambridge University Hospital, Cambridge, UK

\subsection{6/heartjnl-2021-BCS.103}

Introduction The Covid-19 pandemic has put a considerable strain on procedural waiting lists, with the majority of elective outpatient work cancelled during the pandemic. With the vaccination programme and reducing infection levels, attention is turning to addressing these waiting lists. One procedure that was affected was the removal of implantable loop recorders (ILRs). Manufacturers recommend that ILRs are removed at the point of battery depletion (usually 3-4 years), if they have not already been removed due to a positive finding or patient preference. There is little evidence in the literature regarding late complications with ILRs, and we therefore wished to examine what patients' thoughts would be about keeping the ILR in for a longer period of time.

Methods Patients awaiting ILR explantation and those who had undergone explantation, were identified. A retrospective review of the notes was used to get demographic and clinical data. Both groups of patients were contacted, and a questionnaire used to gain an understanding of patients' experience and expectations.

Results Prior to the Covid-19 pandemic, 60 patients who had undergone ILR explantation were identified. A total of 22 responded to our questionnaire (table 1). The majority $(86 \%)$ were happy to have their ILR removed, although a smaller majority (59\%) would also have been happy to have had the device kept in, were it felt to be safe. Very few patients felt a tangible difference as a result of having the ILR removed (14\%) and no patients were worried about the waiting time prior to Covid-19. Of 77 patients currently awaiting explant, 30 responded to our questionnaire (table 1), with $70 \%$ not being concerned by the wait for removal. This is likely aided by the fact that $80 \%$ of patients had no day to day symptoms as a result of the ILR. Half of the patients however, would be concerned about not having the ILR removed, predominantly due to mild discomfort or concerns regarding the presence of a battery. Across both groups $(n=52)$ only 8 patients were concerned about the risk of coming into the hospital for the procedure, with patients commenting that the stringent regulations that hospitals had employed combined with the vaccination programme, gave them significant confidence in attending for outpatient procedures.
Abstract 104 Table 1 Summary of characteristics for each group of patients

\begin{tabular}{lll}
\hline & Waiting list & Explanted \\
\hline $\mathrm{N}$ & 30 & 22 \\
Male (\%) & $17(57 \%)$ & $10(45 \%)$ \\
Age/years & $56.3 \neg\urcorner \underline{14.9}$ & $60.6 \pm 16.3$ \\
Average duration of ILR / years & $4.3+1.53$ & $2.1+1.28$ \\
Average wait from time of listing / years & $1.21+\underline{0.40}$ & - \\
Indication for ILR implant & & $6(27 \%)$ \\
$\quad$ Arrhythmia (\%) & $4(13 \%)$ & $9(41 \%)$ \\
$\quad$ Stroke (\%) & $10(33 \%)$ & $7(32 \%)$ \\
$\quad$ Syncope (\%) & $16(53 \%)$ & $3(14 \%)$ \\
Indication for ILR explant & & $10(45 \%)$ \\
$\quad$ Battery deplete (\%) & $16(53 \%)$ & \\
$\quad$ Arrhythmia detected (\%) & $4(13 \%)$ & \\
\hline
\end{tabular}

Abstract 104 Table 2 Summary of questionnaire responses

\begin{tabular}{lll}
\hline Was the patient concerned about the waiting time? & 9 & $0(0 \%)$ \\
Did the ILR result in a day to day on the patient? & $\begin{array}{l}\text { (30\%) } \\
\text { Did the explant lead to a tangible change for the patient? }\end{array}$ & $(20 \%)$ \\
& - & 3 \\
Would the patient be happy to keep the ILR in? & 15 & 13 \\
& $(14 \%)$ & $(59 \%)$ \\
If not happy, is this due to discomfort? & 7 & 2 \\
If not happy, is this due to concerns about the device/battery itself? & 4 & 0 \\
Was the patient concerned about attending hospital due to the & $1(3 \%)$ & 7 \\
pandemic/lockdown? & & $(32 \%)$ \\
\hline
\end{tabular}

Conclusions This study found that if patients are reassured about the safety of keeping an implantable loop recorder in, and do not suffer any discomfort or symptoms as a result of the device, they are happy both to wait longer for device removal, or even not have it removed at all. In the context of the current pandemic, more thought should perhaps be given to patient guided removal times, especially in cases of removals performed for battery depletion.

Conflict of Interest Nil

\section{EVALUATING LONG TERM PERFORMANCE OF MICRA VA LEADLESS PACEMAKER}

${ }^{1}$ Omar Shaikh, ${ }^{2}$ Sandra Silva, ${ }^{2}$ Berkay Karahacioglu, ${ }^{2}$ Patrick Heck, ${ }^{2}$ David Begley, ${ }^{2}$ Claire Martin. ${ }^{1}$ Papworth, Bedford, UK; ${ }^{2}$ Royal Papworth NHS Foundation Trust

\subsection{6/heartjnl-2021-BCS.104}

Background The Micra VA (leadless) pacemaker was approved by the FDA for use in 2016. Whilst initial studies evaluated the safety and efficacy of the device for a mean follow up of 12 months, very few studies have evaluated complications and device function beyond 1 year.Objective: To evaluate the short and long term complication rate and device function associated with the Micra VA (leadless) pacemaker at our centre. 